\title{
Impacto do ecocardiograma transesofágico intraoperatório na mortalidade em cirurgia de revascularização do miocárdio com circulação extracorpórea
}

\section{Impact of intraoperative transesophageal echocardiography in mortality in coronary artery bypass grafting}

Marcello Fonseca Salgado Filho'; Nubia Verçosa; ismar lima Cavalcanti ${ }^{3}$; Leonardo Augusto Miana4; Cleber Macharet de Souza ${ }^{5}$; Eduardo Borato ${ }^{6}$; Izabela Paltoto ${ }^{7}$

\section{R E S U M O}

\begin{abstract}
Objetivo: avaliar as taxas de mortalidade e morbidade de doentes submetidos à revascularização do miocárdio (RVM) com circulação extracorpórea (CEC) que utilizaram rotineiramente o ecocardiograma transesofágico intraoperatório (ETEio). Métodos: estudo retrospectivo, observacional com avaliação de prontuários de 360 doentes no período entre abril de 2010 a abril de 2012 . Foram analisados: idade, peso, altura sexo, EUROscore, diabete melito, fração de ejeção e artérias acometidas. Os desfechos foram compilados no intra e no pós-operatório (infarto do miocárdio, acidente vascular cerebral, disfunção renal, hemodiálise, fibrilação atrial, tempo de internação no centro de tratamento intensivo). Resultados: foram incluídos 53 doentes, com 27 recebendo a monitoração. Foram excluídos 307 porque não foram operados pela mesma equipe cirúrgica. Os dois grupos foram homogêneos quanto a idade, peso e sexo, porém, a fração ejeção foi menor no grupo que recebeu o ecotransesofágico (G ETEio: 56,3\%; G Não ETEio: $65,9 \% \pm 11 ; p=0,01$ ). Nos doentes em que não foi utilizado o ETEio, a mortalidade foi maior (G ETEio: $0 \%$ e $G$ Não ETEio: $7,6 \% ; p=0,01)$. Não houve diferença significativa entre os grupos quanto à incidência de acidente vascular encefálico, infarto agudo do miocárdio, fibrilação atrial aguda e lesão renal. Conclusão: a utilização do ecocardiograma transesofágico intraoperatório em pacientes submetidos à revascularização do miocárdio, com circulação extracorpórea, diminuiu a mortalidade perioperatória; orientou quanto a utilização dos fármacos inotrópicos e vasodilatadores e contribuiu para uma melhor evolução dos doentes.
\end{abstract}

Descritores: Ecocardiografia transesofagiana. Procedimentos cirúrgicos cardíacos. Circulação extracorpórea. Cardiotônicos. Vasodilatadores.

\section{INTRODUÇÃO}

\begin{abstract}
A primeira operação de revascularização do miocárdio (RVM) foi realizada em 1967 pelo Dr. Willian Longmire, que fez a anastomose da veia safena esquerda à artéria coronária direita'. Com a inovação tecnológica e aprimoramento técnico, a cirurgia cardíaca difundiu-se mundialmente. Hoje em dia, entre as operações cardíacas, a revascularização do miocárdio é o procedimento cirúrgico mais realizado².

Dentre as melhorias técnicas, podemos ressaltar as melhores estratégias de proteção miocárdica, melhores condutas de manejo da artéria mamária interna, avanços na monitoração, destacando-se o ecocardiograma transesofágico intraoperatório (ETEio) e o desenvolvimen-
\end{abstract}

to de dispositivos para suporte ventricular ${ }^{3-5}$ e a descoberta de novos fármacos. Observa-se, com isso, uma menor incidência de mortalidade e complicações dos pacientes submetidos à revascularização do miocárdio com circulação extracorpórea $(\mathrm{CEC})^{3-5}$.

A taxa de mortalidade mundial intra-hospitalar varia de $3,2 \%^{3}$ a $10 \%{ }^{6}$. Dentre os fatores de risco que mais contribuem para a mortalidade perioperatória destacam-se o diabete melito insulino dependente, a insuficiência renal crônica, com necessidade de hemodiálise, as operações de emergência, o choque cardiogênico e as reoperacões 6 .

Uma das complicações perioperatórias mais temida da revascularização do miocárdio é o infarto perioperatório. Dentro da classificação atual do infarto agu-

Programa de Pós-Graduação em Ciências Cirúrgicas do Departamento de Cirurgia da Faculdade de Medicina da Universidade Federal do Rio de Janeiro. Trabalho realizado na Santa Casa de Misericórdia de Juiz de Fora, Minas Gerais.

1. Coordenador do Curso de Ecocardiografia Intraoperatória da Sociedade Brasileira de Anestesiologia; 2. Professora Associada do Departamento de Cirurgia da Faculdade de Medicina da Universidade Federal do Rio de Janeiro; 3. Professor Adjunto da Universidade Federal Fluminense; 4. Professor Adjunto da Universidade Federal de Juiz de Fora; 5. Membro do Comitê de Anestesia Cardiovascular da Sociedade Brasileira de Anestesiologia; 6. Cardiologista da Santa Casa de Misericórdia de Juiz de Fora/MG; 7. Enfermeira do Hospital Maternidade Monte Sinai, Juiz de Fora/Minas Gerais. 
do do miocárdio, o infarto intraoperatório é classificado como tipo I (ruptura plaquetária) e tipo II (desequilíbrio entre oferta e demanda de oxigênio) ${ }^{7}$.

Durante a realização da revascularização do miocárdio, a presença de uma nova disfunção segmentar ventricular diagnosticada pelo ETEio tem $100 \%$ de valor preditivo positivo, além de localizar a artéria coronariana acometida ${ }^{7}$. O ETEio, também, orienta a reposição volêmica e o uso de fármacos inotrópicos e vasodilatadores durante o intraoperatório, o que resulta em menor mortalidade hospitalar ${ }^{6,7}$.

Contudo, O'Brien et al. ${ }^{8}$ demonstraram que os doentes submetidos à cirurgia cardíaca que apresentavam maior EuroSCORE (avaliação européia do risco cardíaco operatório), menor classe funcional pela New York Heart Association (NYHA) e que, utilizaram rotineiramente o ETEio, o hemoconcentrador (usado para ultrafiltração sanguínea e remoção do excesso de fluido) e foram mais hemotransfundidos, apresentaram um maior índice de mortalidade.

O objetivo deste estudo foi avaliar as taxas de mortalidade e morbidade de doentes submetidos à revascularização do miocárdio (RVM) com circulação extracorpórea (CEC) que utilizaram rotineiramente o ETEio e comparar com àqueles que não receberam esse tipo de monitoração.

\section{MÉTODOS}

Após aprovação pelo Comitê de Ética em Pesquisa da Santa Casa de Misericórdia de Juiz de Fora, Minas Gerais (Parecer n 39525/2012) foi realizada a análise retrospectiva dos prontuários de 360 pacientes submetidos à RVM com CEC no período de abril de 2010 a abril de 2012.

Foram incluídos no estudo, doentes com idade acima de 40 anos, de ambos os sexos, com doença obstrutiva coronariana, em uso de betabloqueadores com ritmo sinusal no eletrocardiograma, fração de ejeção maior que $35 \%$ e hematócrito maior que $30 \mathrm{~g} / \mathrm{dl}$, submetidos à RVM com CEC pela mesma equipe.

Os critérios de exclusão foram: operações de RVM sem CEC, cirurgia de emergência (com menos de seis horas de cateterismo cardíaco), operações combinadas (operações valvulares, aneurismectomia de ventrículo esquerdo, operações na aorta, endarterectomia carotídea); instabilidade hemodinâmica (uso de inotrópicos apos indução anestésica até o início da (EC); precordialgia e/ ou alteração eletrocardiográfica antes da indução anestésica; pacientes com significativa lesão valvular (moderada a grave, insuficiência ou estenose), em uso de balão intra-aórtico (BIA) e creatinina plasmática maior que $2 \mathrm{mg} / \mathrm{dl}$ no período pré-operatório.

Todas as operações foram realizadas pela mesma equipe e a avaliação pelo ETEio foi realizado por um anestesiologista experiente. Os exames ecocardiográficos foram realizados por meio do aparelho Vivid ${ }^{\circledR}$ (GE Medical System, Israel), segundo os critérios da Sociedade Americana de Anestesiologia Cardiovascular9,10.

Foram anotados a classificação do EuroSCORE, o ASA, a fração de ejeção, a presença de diabete melito, o número de artérias coronárias acometidas, presença de infarto perioperatório, insuficiência cardíaca, acidente vascular encefálico, disfunção renal, fibrilação atrial aguda perioperatória, tempo (minutos) de CEC, número de anastomoses coronarianas e tempo (dias) de internação no centro de tratamento intensivo (CTI).

Os grupos foram classificados quanto ao uso ou não de ETEio. Todos os pacientes do grupo ETEio foram submetidos à RVM com CEC e monitorados com o ecotransesofágico desde a indução da anestesia até o término do procedimento anestésico.

Os pacientes do grupo Não-ETEio foram submetidos aos mesmos procedimentos cirúrgicos, porém não receberam essa monitoração.

O pós-operatório imediato foi realizado no CTI, onde todos os doentes, de ambos os grupos, receberam as mesmas avaliações hemodinâmicas e tratamentos clínicos.

Para a análise da evolução dos doentes, adotaram-se os desfechos, observados no intra e pós-operatórios até a alta hospitalar: 1) mortalidade intra-hospitalar; 2) infarto agudo do miocárdio perioperatório, (diagnosticado quando surgia uma nova onda Q no eletrocardiograma ou elevação do segmento ST - Código de Minesota) ${ }^{11,12 ;}$;) quando as enzimas cardíacas (creatinoquinase fração MB (CK-MB) forem cinco vezes acima dos valores basais) $\left.{ }^{12} ; 4\right)$ insuficiência cardíaca perioperatória, definida pela necessidade de utilização de suporte ventricular (membrana extracorpórea de oxigenação - otrópicas ou vasopressoras por mais que 48 horas $^{11}$; ECMO); 5) uso de balão intraaórtico (BIA); 6) utilização de drogas inotrópicas ou vasopressoras por mais que 48 horas; 7 ) acidente vascular encefálico perioperatório diagnosticado por avaliação clínica e confirmado por exame de imagem (tomografia computadorizada de crânio (TCC) ou ressonância nuclear magnética (RNM) de crânio ${ }^{11}$; 8) disfunção renal perioperatória - quando a creatinina plasmática era maior ou igual a $2 \mathrm{mg} / \mathrm{dl}$, acompanhada por um acréscimo de $0,7 \mathrm{mg} / \mathrm{dl}$ dos valores basais ${ }^{11}$; falência renal foi definida quando a disfunção renal requeria hemodiálise ${ }^{11}$; 9) tempo total de CEC; 10) número de anastomoses coronarianas; 11) incidência de fibrilação atrial aguda; e 12) tempo de internação no CTI.

A análise do ETEio foi realizada no período préCEC (após a indução anestésica e antes da incisão cirúrgica) e no período pós-CEC, durante o desmame de circulação extra-corpórea, dez minutos após o término da infusão venosa da protamina e após o fechamento do esterno.

O estudo ecocardiográfico avaliava os seguintes parâmetros: 1) análise da função global do ventrículo esquerdo (VE) e direito (VD); 2) análise das válvulas mitral, 
tricúspide, aórtica e pulmonar; 3) débito cardíaco do VE; 4) índice de Tei (avaliação da função ventricular) do VE; 5) padrão diastólico do VE ${ }^{10-13}$.

Os dados paramétricos foram analisados pelo teste t de Student. Os dados não paramétricos pelo teste Mann-Whitney e os dados categóricos pelo teste Qui-quadrado ou teste de Fisher. O programa utilizado foi o GraphPad Prism versão 5.00 para Mac OS X, GraphPad Software, San Diego California USA, www.graphpad.com. A significância estatística para valores de $p<0,05$. Os resultados estão apresentados como média \pm desvio padrão.

O cálculo para 25 pacientes por grupo, perfazendo um total de 50 pacientes, corresponde a um valor de confiança igual a 6 para $95 \%$ de probabilidade.

\section{RESULTADOS}

Dos 360 prontuários analisados dos pacientes operados, 289 foram excluídos porque não foram submetidos à RVM pela mesma equipe (anestesista/cirurgião/ perfusionista). Dos 71 pacientes restantes, três apresentaram fração de ejeção menor que 35\%, dez foram submetidos à operações combinadas e cinco, apresentaram creatinina plasmática maior que $2 \mathrm{mg} / \mathrm{dl}$. No total, 53 pacientes foram submetidos à análise estatística: 27 (59,9\%) deles pertenceram ao grupo ETEio e $26(49,1 \%)$ ao grupo Não ETEio (Figura 1).

Na tabela 1 estão explicitados os valores demográficos e os fatores de risco de ambos os grupos. Não houve diferença estatística entre os grupos, exceto a fração de ejeção pré-operatória que foi menor no grupo que utilizou o ETEio $(p=0,01)$.

A figura 2 apresenta o resultado da mortalidade e sobrevida durante o período de internação hospitalar entre os dois grupos. Não houve óbito no grupo ETEio $(p=0,001)$. O grupo Não-ETEio apresentou 7,6\% de mortalidade.

Não houve diferença entre os desfechos estudados com os dois grupos no período intraoperatório e pós-operatório até a alta hospitalar. O número de anastomoses coronarianas, o tempo de CEC e número de dias no CTI foram semelhantes em ambos os grupos (Tabela 2).

\section{DISCUSSÃO}

Os resultados deste estudo retrospectivo observacional demonstraram que os pacientes submetidos à RVM com CEC que não utilizaram o ETEio como monitoração de rotina, apresentaram uma maior mortali-

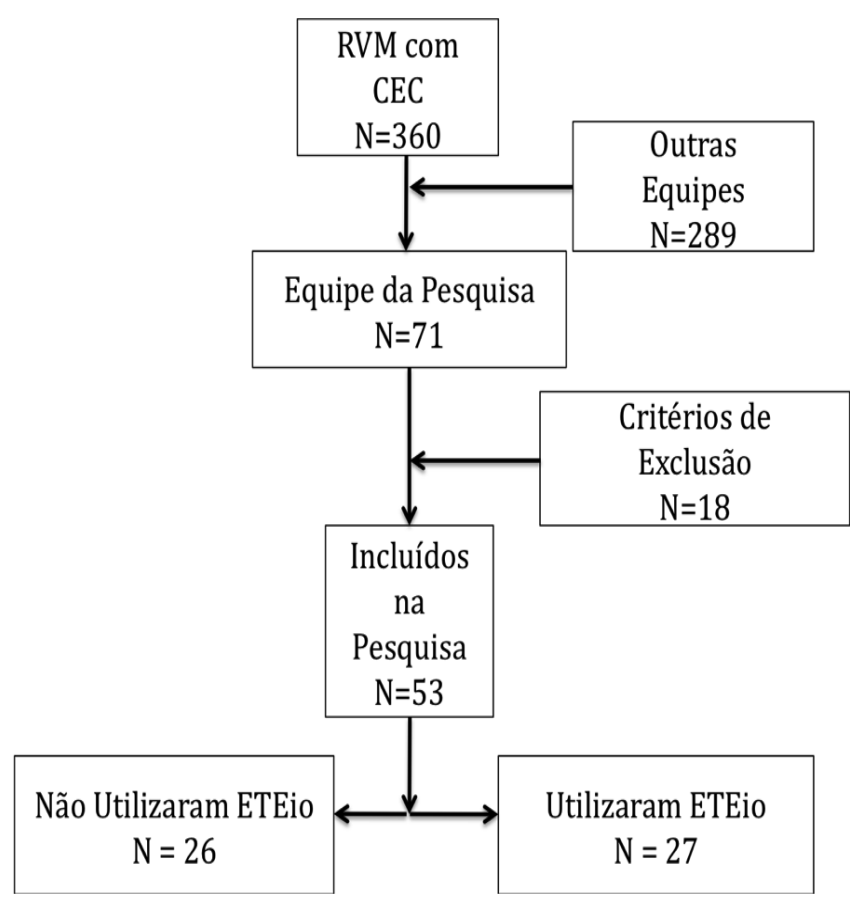

Figura 1 - Desenho do estudo demonstrando o número total de pacientes submetidos à revascularização do miocárdio (RVM) com circulação extra-corpórea (CEC), pacientes excluídos, e total de pacientes incluídos na pesquisa e analisados no grupo Ecotransesofágico intraoperatória (ETEio) e Não Ecotransesofágico intraoperatório (Não ETEio).

Tabela 1 - Características demográficas e fatores de risco dos pacientes durante a avaliação pré-anestesica.

\begin{tabular}{lcccc}
\hline Dados & Sem ETEio & Com ETEio & $p$ Valor \\
\hline Total de Pacientes, N (\%) & $26 \quad(49,1 \%)$ & 27 & $(50,9 \%)$ & 0,88 \\
Idade (Anos) & $63,1 \pm 11$ & $61,2 \pm 8,8$ & 0,73 \\
Peso (Kg) & $72,8 \pm 9,8$ & $68,6 \pm 11$ & 0,05 \\
Altura (Cm) & $166,5 \pm 6,5$ & $163 \pm 10,5$ & 0,15 \\
Genero Masculino, N (\%) & $19 \quad(73 \%)$ & $16 \quad(59 \%)$ & 0,05 \\
Euroescore (Pontos) & $6,9 \pm 6,1$ & $7,0 \pm 6,6$ & 0,89 \\
Diabetes Mellitus, N (\%) & $6(30 \%)$ & $7(35 \%)$ & 0,5 \\
Fracao de Ejecao (\%) & $65,9 \pm 11$ & $56,3 \pm 14$ & $0,01^{*}$ \\
Arterias Coronarianas Acometidas, $n$ & $3,3 \pm 0,6$ & $3,4 \pm 0,9$ & 0,85 \\
\hline
\end{tabular}

* - significância estatística, $p<0,05$ entre as medias dos dois grupos pelo teste $t$ de student. 
Tabela 2 - Tempo de CEC, número de anastomoses coronarianas e desfechos dos pacientes no período intra-operatório e no pós-operatório até a alta hospitalar.

\begin{tabular}{|c|c|c|c|}
\hline Dados & Sem ETEio & Com ETEio & $p$ Valor \\
\hline CEC (min) & $91 \pm 25$ & $81,2 \pm 23,6$ & 0,33 \\
\hline Anastomoses Coronarianas, $\mathrm{N}$ & $3,3 \pm 0,7$ & $3 \pm 0,9$ & 0,08 \\
\hline lam Pós-operatório, N (\%) & $4 \quad(15 \%)$ & $2 \quad(7 \%)$ & 0,11 \\
\hline Acidente Vascular Encefalico no Pós-operatório, N (\%) & $(3,8 \%)$ & $(3,9 \%)$ & 1,00 \\
\hline Disfunção Renal Aguda no Pós-operatório, N (\%) & $(15 \%)$ & $(18 \%)$ & 0,7 \\
\hline Hemodiálise (\%) & $(0 \%)$ & $(0 \%)$ & 1,00 \\
\hline Fibrilação Atrial Aguda no Pós-operatório (\%) & $5 \quad(19 \%)$ & $3 \quad(11 \%)$ & 0,16 \\
\hline Tempo de Intenacao no CTI (dias) & $3,3 \pm \quad 0,6$ & $3,4 \pm \quad 1,4$ & 0,78 \\
\hline
\end{tabular}

CEC-Cirulação extra-corpórea, IAM - Infarto agudo do miocárdio, CTI - Centro de tratamento intensivo. P>0,05 para todos os dados estudados.

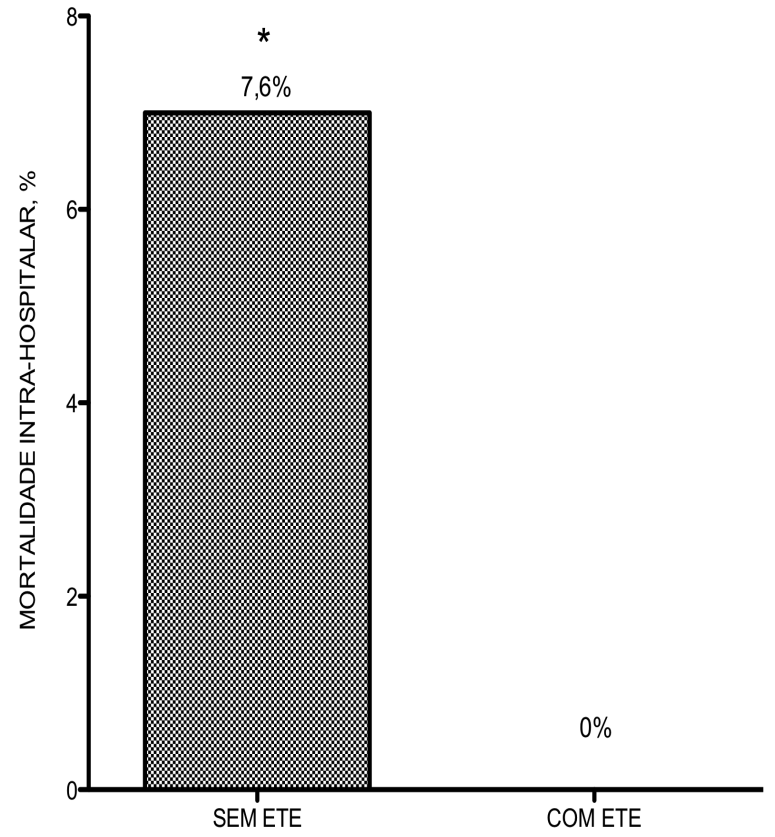

Figura 2 - Incidência de mortalidade intra-hospitalar entre o grupo Não ETE e o grupo ETE.

dade do que o grupo de pacientes que utilizaram o ETEio rotineiramente, $7,6 \%$ e $0 \%$, respectivamente.

No Brasil, ainda não existe nenhum estudo que tenha avaliado a mortalidade e morbidade em cirurgia de RVM com CEC comparando a utilização ou não do ETEio.

Dos doentes que foram a óbito, um apresentou AVE isquêmico que evoluiu com pneumonia e sepsis e o outro apresentou choque cardiogênico.

Estudos multicêntricos que analisaram a mortalidade e morbidade em doentes monitorados por meio de cateter de artéria pulmonar (padrão ouro para avaliação da função ventricular em pacientes submetidos à RVM) demonstraram que os pacientes que utilizaram este tipo de monitoração apresentaram uma maior incidência de disfunção orgânica e mortalidade durante a internação hospitalar $r^{3,11,14}$.
O ETEio é utilizado nos Estados Unidos da América em operações cardíacas desde a década de $80^{3,15}$ e Bergquist et al. ${ }^{16}$, em 1996, demonstraram em um estudo aleatório prospectivo que, nos pacientes em que se utilizou o ETEio rotineiramente em cirurgias cardíacas, houve uma menor mortalidade e morbidade quando comparado com o grupo em que não se usou o ETEio. Outro estudo, com 850 pacientes, concluiu que a utilização do ETEio diminuiu a mortalidade e a morbidade dos doentes submetidos a procedimentos com $\mathrm{CEC}^{4}$ e que o ETEio deve ser usado, rotineiramente, em operações cardiacas ${ }^{3}$.

No presente estudo, o grupo que utilizou o ETEio apresentava uma menor fração de ejeção no período préoperatório. Desta forma, esperava-se uma maior morbidade e mortalidade neste grupo, porque os pacientes coronariopatas apresentam uma maior incidência de mortalidade à medida que a função ventricular esquerda vai se deteriorando ${ }^{6}$. Porém, os resultados encontrados mostraram que o grupo ETEio, apesar de ter uma fração de ejeção menor no pré-operatório, apresentou menor mortalidade intra-hospitalar. O mesmo resultado foi demonstrado por Savage et al. ${ }^{3}$ e Silva et al. ${ }^{4}$.

Como a função ventricular esquerda consiste na resultante do enchimento e esvaziamento do ventrículo esquerdo, tanto a função sistólica quanto a diastólica, podem comprometer de forma isolada o desempenho cardíaco (avaliação sisto-diastólica) ${ }^{13}$. Desta forma, utilizando o ETEio, é possível orientar a melhor terapêutica, otimizando a função sistólica e diastólica do VE durante a cirurgia de revascularização do miocárdio $3,4,16$.

Demosntrou-se que a utilização do ETEio na revascularização do miocárdio pode otimizar a terapêutica de reposição volêmica em até $47 \%$ dos casos. Além disso, o ETEio isoladamente, foi o monitor que mais influenciou positivamente na tomada de decisões quanto ao uso de inotrópicos, vasodilatadores e reposição volêmica ${ }^{3,4,16}$.

Segundo a Sociedade Americana de Anestesia Cardiovascular, mais de $72 \%$ dos anestesiologistas que trabalham com anestesia cardíaca usam, rotineiramente, o ETEio. Além disso, o ETEio apresenta 12,8\% de novas in- 
formações e pode mudar a conduta cirúrgica em até $48 \%$ dos $\operatorname{casos}^{3,4,16}$.

No presente estudo, apesar de não ter havido diferença na morbidade entre os dois grupos, pode-se observar uma tendência a menor incidência de infarto agudo do miocárdio perioperatório e fibrilação atrial perioperatória no grupo ETEio. Estes resultados também foram publicados por Savage et al., em um estudo prospectivo com 82 pacientes ${ }^{3}$.

O presente estudo apresenta as seguintes limitações: o pequeno número de pacientes avaliados e a au- sência de aleatorização da amostra estudada não permite extrapolar esta análise para a população em geral.

Para confirmar estes resultados, seria importante a realização de outros estudos aleatorizados e prospectivos com um número maior de pacientes.

A utilização do ecocardiograma transesofágico intraoperatório em pacientes submetidos à cirurgia de revascularização do miocárdio, com circulação extracorpórea, diminuiu a mortalidade perioperatória, orientou quanto à utilização dos fármacos inotrópicos e vasodilatadores e contribuiu para uma melhor evolução dos doentes.

\title{
A $B$ S $S$ T R A C T
}

\begin{abstract}
Objective: To evaluate the rates of mortality and morbidity in patients undergoing coronary artery bypass grafting (CABG) with cardiopulmonary bypass (CPB) using routine intraoperative transesophageal echocardiography (ITEE). Methods: We conducted a retrospective, observational review of medical records of 360 patients from April 2010 to April 2012. We analyzed: age, weight, height, gender, EuroSCORE, diabetes mellitus, ejection fraction and number of diseased vessels. Outcomes were compiled in intraoperative and postoperative (myocardial infarction, stroke, renal failure, hemodialysis, atrial fibrillation, length of stay in the intensive care unit). Results: 53 patients were included, with 27 receiving monitoring; we excluded 307 individuals, as they were not operated by the same surgical team. The two groups were homogeneous for age, weight and gender. However, the ejection fraction was lower in the group submitted to ITEE (ITEE group $56.3 \%$ versus Non-ITEE group $65.9 \pm 11 \%, p=0.01$ ). In patients not subjected to ITEE, mortality was higher (ITEE group $0 \%$ versus Non-ITEE group $7.6 \%, p=0.01$ ). There was no significant difference between groups as for the incidence of stroke, myocardial infarction, atrial fibrillation and acute kidney injury. Conclusion: The use of intraoperative transesophageal echocardiography in patients undergoing coronary artery bypass grafting with cardiopulmonary bypass decreased perioperative mortality, warranted the use of inotropic drugs and vasodilators and contributed to a better patient outcome.
\end{abstract}

Key words: Transesophageal echocardiography. Cardiac surgical procedures. Extracorporeal circulation. Cardiotonic. Vasodilators.

\section{REFERÊNCIAS}

1. Favaloro RG. Saphenous vein autograft replacement of severe segmental coronary artery oclusion: operative technique. Ann Thorac Surg. 1968;5(4):334-9.

2. Ricciardi R, Virnig BA, Ogilvie JW Jr, Dahlberg PS, Selker HP, Baxter NN. Volume-outcome relationship for coronary artery bypass grafting in an era of decreasing volume. Arch Surg. 2008;143(4):338-44

3. Savage RM, Lytle BW, Aronson S, Navia JL, Licina M, Stewart WJ, et al. Intraoperative echocardiography is indicated in high-risk coronary artery bypass grafting. Ann Thorac Surg. 1997;64(2):36873; discussion 373-4.

4. Silva F, Arruda R, Nobre A, Mendes M, Lemos A, Gallego J, et al. Impacto da ecocardiografia transesofaìgica intra-operatoiria na cirurgia cardiìaca. Anailise retrospectiva duma seirie de 850 exames. Rev Port Cardiol. 2010;29(9):1363-82

5. Chumnanvej S, Wood MJ, MacGillivray TE, Melo MF. Perioperative echocardiographic examination for ventricular assist device implantation. Anesth Analg. 2007;105(3):583-601.

6. Shahian DM, O'Brien SM, Sheng S, Grover FL, Mayer JE, Jacobs $J P$, et al. Predictors of long-term survival after coronary artery bypass grafting surgery: results from the Society of Thoracic Surgeons Adult Cardiac Surgery Database (the ASCERT study). Circulation. 2012;125(12):1491-500.

7. Subramaniam B, Subramaniam K. Not all perioperative myocardial infarctions can be prevented with preoperative revascularization. Anesthesiology. 2010;112(3):524-6.

8. O'Brien MM, Shroyer AL, Moritz TE, London MJ, Grunwald GK Villanueva $C B$, et al. Relationship between processes of care and coronary bypass operative mortality and morbidity. Med Care. 2004:42(1):59-70.

9. Shanewise JS, Cheung AT, Aronson S, Stewart WJ, Weiss RL, Mark JB, et al. ASE/SCA guidelines for performing a comprehensive intraoperative multiplane transesophageal echocardiography examination: recommendations of the American Society of Echocardiography Council for Intraoperative Echocardiography and the Society of Cardiovascular Anesthesiologists Task Force for Certification in Perioperative Transesophageal Echocardiography. J Am Soc Echocardiogr. 1999;12(10):884-900

10. Salgado Filho MF, Siciliano A, Siciliano A, Oliveira AJ, Salgado J, Palitot I. A importância do ecocardiograma transesofágico na captação do coração para transplante cardíaco. Rev bras anestesiol. 2012:62(2):262-8.

11. Schwann NM, Hillel Z, Hoeft A, Barash P, Möhnle P, Miao Y, et al Lack of effectiveness of the pulmonary artery catheter in cardiac surgery. Anesth Analg. 2011;113(5):994-1002.

12. Mangano DT, Browner WS, Hollenberg M, London MJ, Tubau JF, Tateo IM. Association of perioperative myocardial ischemia with cardiac morbidity and mortality in men undergoing noncardiac surgery. The Study of Perioperative Ischemia Research Group. N Engl J Med. 1990;323(26):1781-8.

13. Matyal R, Skubas NJ, Shernan SK, Mahmood F. Perioperative assessment of diastolic dysfunction. Anesth Analg. 2011:113(3):449-72.

14. Binanay C, Califf R, Hasselblad V, O'Connor CM, Shah MR, Sopko $G$, et al. Evaluation study of congestive heart failure and pulmonary artery catheterization effectiveness: the ESCAPE trial. JAMA 2005;294(13):1625-33. 
15. Salgado Filho MF, Siciliano A, Diego LA, Miana LA, Salgado J. O ecocardiograma transesofágico na cirurgia de Ross. Rev bras anestesiol. 2011;61(3):344-50

16. Bergquist BD, Bellows WH, Leung JM. Transesophageal echocardiography in myocardial revascularization: II. Influence on Intraoperative decision making. Anesth Analg. 1996;82(6):113945.

Recebido em 02/09/2012

Aceito para publicação em 05/11/2012

Conflito de interesse: nenhum

Fonte de financiamento: nenhuma

\section{Como citar este artigo:}

Salgado Filho MF, Figueiredo NV, Cavalcanti IL, Miana LA, Souza CM, Borato E, Paliot I. Impacto do ecocardiograma transesofágico intraoperatório na mortalidade em cirurgia de revascularização do miocárdio com circulação extracopórea. Rev Col Bras Cir. [periódico na Internet] 2013;40(5). Disponível em URL: http://www.scielo.br/rcbc

Endereço para correspondência:

Marcello Fonseca Salgado Filho

E-mail: mfonsecasalgado@hotmail.com 